\title{
Insensitizing Controls for a Large-Scale Ocean Circulation Model
}

Enrique FERNANDEZ-CARA ${ }^{\mathrm{a}}$, Galina C. GARCIA ${ }^{\mathrm{b}}$ and Axel OSSES ${ }^{\mathrm{c}}$

${ }^{a}$ Dpto. E.D.A.N., Universidad de Sevilla, Aptdo. 1160, 41080 Sevilla, Spain.

b Dpto. Ingeniería Matemática, Universidad de Concepción, Casilla 160-C, Concepción and Centro de Modelamiento Matemático, UMR 2071 CNRS-Universidad de Chile, Santiago, Chile.

c Dpto. Ingeniería Matemática, Universidad de Chile, Casilla 170/3 Correo 3, Santiago and Centro de Modelamiento Matemático, UMR 2071 CNRS-Universidad de Chile, Santiago, Chile.

\begin{abstract}
We consider here a linear quasi-geostrophic ocean model. We look for controls insensitizing (resp. $\varepsilon$-insensitizing) an observation function of the state. The existence of such controls is equivalent to a null controllability property (resp. an approximate controllability property) for a cascade Stokes-like system. Under reasonable assumptions on the spatial domains where the observation and the control are performed, we are able to prove these properties.
\end{abstract}

\section{Introduction and main result}

Let $\Omega$ be a nonempty open bounded connected subset of $\mathbb{R}^{2}$, with boundary $\Gamma$ of class $\mathcal{C}^{2}$ and outwards unit normal vector $\nu=\nu(x)$. Let $\omega$ be a nonempty open subset of $\Omega, T>0, Q=$ $\Omega \times(0, T)$ and $\Sigma=\Gamma \times(0, T)$. We consider a linear quasi-geostrophic ocean model [9, 10], described by the following equations

$$
\left\{\begin{array}{l}
u_{t}-A \Delta u+\gamma u+\left(f_{0}+\beta x_{2}\right) \mathrm{k} \wedge u+\frac{1}{\rho_{0}} \nabla p=\mathcal{T}+h 1_{\omega} \quad \text { in } Q, \\
\operatorname{div} u=0 \text { in } Q \\
u=0 \text { on } \Sigma, \\
\left.u\right|_{t=0}=u_{0}+\tau \hat{u}_{0} \text { in } \Omega .
\end{array}\right.
$$

Here, $u=u(x, t)$ and $p=p(x, t)$ are the velocity field and the pressure of the fluid. In this model, $A$ is the horizontal eddy viscosity coefficient, $\gamma$ is the bottom friction coefficient, $\rho_{0}$ is the 
fluid density and $\left(f_{0}+\beta x_{2}\right) \mathrm{k} \wedge u$ is the Coriolis term, with $\mathrm{k} \wedge u=\left(-u_{2}, u_{1}\right)$. In the right hand side, $1_{\omega}$ denotes the characteristic function of $\omega$ and $\mathcal{T}$ is a given source. The term $\tau \hat{u}_{0}$, where $\tau \in \mathbb{R}$, represents a small unknown perturbation of the initial condition $u_{0}$ and $h=h(x, t)$ is a control term to be determined.

Notice that the Coriolis force is modelled by a zero order coupling term in the equations. It introduces a different behavior of the system depending on the direction in space. In order to simplify the presentation of the results, we will assume that $A=\gamma=f_{0}=\beta=\rho_{0}=1$.

Let us recall the classical spaces

$$
H=\left\{v \in L^{2}(\Omega)^{2}: \operatorname{div} v=0 \text { in } \Omega, v \cdot \nu=0 \text { on } \Gamma\right\}
$$

and

$$
V=\left\{v \in H_{0}^{1}(\Omega)^{2}: \operatorname{div} v=0 \text { in } \Omega\right\} .
$$

Assume that $u_{0}, \tau \hat{u}_{0} \in H$, with $\left\|\hat{u}_{0}\right\|_{0, \Omega}=1, \mathcal{T} \in L^{2}(Q)$ and $h \in L^{2}(\omega \times(0, T))$. Then problem (1) possesses exactly one solution $(u, p)$, with $u \in L^{2}(0, T ; V) \cap H^{1}\left(0, T ; V^{\prime}\right) \cap \mathcal{C}([0, T] ; H)$ and $p \in \mathcal{D}^{\prime}\left(0, T ; L_{0}^{2}(\Omega)\right)$. This can be easily proved by adapting the arguments of [11].

Let $\mathcal{O}$ be a non-empty subset of $\Omega$ and let us introduce the functional $\Phi$, with

$$
\Phi(u)=\frac{1}{2} \int_{0}^{T} \int_{\mathcal{O}}|u(x, t)|^{2} d x d t .
$$

The notion of insensitizing control was introduced by J.L. Lions in [8]. In the context of (1), it can be formulated as follows:

Definition 1.1 We say that the control $h$ is insensitizing $\Phi$ if

$$
\left.\frac{d}{d \tau} \Phi(u)\right|_{\tau=0}=0 \quad \forall \hat{u}_{0} \in H,\left\|\hat{u}_{0}\right\|_{0, \Omega}=1 .
$$

On the other hand, we say that $h$ is $\varepsilon$-insensitizing $\Phi$ if

$$
\left|\frac{d}{d \tau} \Phi(u)\right|_{\tau=0} \mid \leqslant \varepsilon \quad \forall \hat{u}_{0} \in H, \quad\left\|\hat{u}_{0}\right\|_{0, \Omega}=1 .
$$

Of course, in (3) and (4), $u$ is, together with $p$, the unique solution to (1).

The existence of insensitizing and $\varepsilon$-insensitizing controls for linear and semilinear heat equations has been studied in $[1,2,12]$. In this Note, we will be concerned with this question in the case of the previous Stokes-like system.

As in the previous references, we will impose the following geometrical hypothesis:

$$
\omega \cap \mathcal{O} \neq \emptyset \text {. }
$$

The main results of this Note are the following:

Theorem 1.2 Let $T>0$ and assume that (5) is satisfied. Then, for each $\varepsilon>0$, there exists $a$ control $h \in L^{2}(\omega \times(0, T))$ which is $\varepsilon$-insensitizing $\Phi$.

Theorem 1.3 Under the assumptions of Theorem 1.2, if we also have $u_{0}=0$, there exists $a$ constant $M(\Omega, \omega, \mathcal{O}, T)>0$ such that, whenever

$$
\int_{0}^{T} \int_{\Omega} \exp \left(M / t^{4}\right) \mathcal{T}^{2} d x d t<\infty
$$

there exists a control $h \in L^{2}(\omega \times(0, T))$ which is insensitizing $\Phi$. 


\section{Proof of the main results}

Let us first give a characterization of the insensitivity (resp. $\varepsilon$-insensitivity) properties in terms of the null controllability (resp. approximate controllability) of an appropriate cascade system. This is obtained by differentiating $\Phi$ with respect to the parameter $\tau$ and following a standard argument, see $[1,12]$ :

Proposition 2.1 Let $(\bar{u}, \bar{p}, q, \pi)$ be the solution of the following system:

$$
\left\{\begin{array}{l}
\bar{u}_{t}-\Delta \bar{u}+\bar{u}+\left(1+x_{2}\right) k \wedge \bar{u}+\nabla \bar{p}=\mathcal{T}+h 1_{\omega} \text { in } Q, \\
-q_{t}-\Delta q+q-\left(1+x_{2}\right) k \wedge q+\nabla \pi=\bar{u} 1_{\mathcal{O}} \text { in } Q, \\
\operatorname{div} \bar{u}=0, \quad \operatorname{div} q=0 \quad \text { in } Q, \\
\bar{u}=q=0 \quad \text { on } \Sigma, \\
\left.\bar{u}\right|_{t=0}=u_{0},\left.\quad q\right|_{t=T}=0 \quad \text { in } \Omega .
\end{array}\right.
$$

Then the control h is insensitizing $\Phi$ (resp. $\varepsilon$-insensitizing $\Phi)$ if and only if

$$
\left.q\right|_{t=0}=0 \quad\left(\text { resp. }\left\|\left.q\right|_{t=0}\right\|_{0, \Omega} \leqslant \varepsilon\right) .
$$

In general, for linear problems, it is well known that approximate controllability is equivalent to a unique continuation property of the associate adjoint system and that exact controllability reduces to suitable observability estimates. We will now present the main steps in the proofs of Theorems 1.2 and 1.3, which are inspired by these general principles. Full versions of the proofs will appear in a forthcoming paper.

Proposition 2.2 Assume (5). Let $(z, r, \phi, \theta)$ be the solution to

$$
\left\{\begin{array}{l}
-z_{t}-\Delta z+z-\left(1+x_{2}\right) k \wedge z+\nabla r=\phi 1_{\mathcal{O}} \text { in } Q, \\
\phi_{t}-\Delta \phi+\phi+\left(1+x_{2}\right) k \wedge \phi+\nabla \theta=0 \quad \text { in } Q, \\
\operatorname{div} z=0, \quad \operatorname{div} \phi=0 \text { in } Q, \\
z=0, \quad \phi=0 \text { on } \Sigma, \\
\left.z\right|_{t=T}=0,\left.\quad \phi\right|_{t=0}=\phi_{0} \text { in } \Omega,
\end{array}\right.
$$

where $\phi_{0} \in V$. Then, if $z=0$ in $\omega \times(0, T)$, we necessarily have $z \equiv \phi \equiv 0$ and $\nabla r \equiv \nabla \theta \equiv 0$ in $Q$.

Sketch of the proof: Let us put $\widetilde{\omega}=\omega \cap \mathcal{O}$. We will prove a more general result saying that

$$
\left\{\begin{array}{l}
-z_{t}-\Delta z+z-\left(1+x_{2}\right) k \wedge z+\nabla r=\phi 1_{\mathcal{O}} \quad \text { in } Q \\
\phi_{t}-\Delta \phi+\phi+\left(1+x_{2}\right) k \wedge \phi+\nabla \theta=0 \text { in } Q \\
\operatorname{div} z=0, \quad \operatorname{div} \phi=0 \quad \text { in } Q \\
\phi_{1}=0 \text { on } \Sigma_{1},\left.\quad \phi\right|_{t=0} \in V
\end{array}\right.
$$

where $\Sigma_{1}=\left(\Gamma \cap C_{1}\right) \times(0, T), C_{1}=\left\{\left(x_{1}, x_{2}\right) \in \Omega: \exists x_{1}^{0}\right.$ s.t. $\left.\left(x_{1}^{0}, x_{2}\right) \in \widetilde{\omega}\right\}$. Then, if $z=0$ in $\omega \times(0, T)$, we necessarily have $\phi \equiv 0$ in $Q$. We first notice that curl $\phi=0$ in $\widetilde{\omega} \times(0, T)$. Then, applying the curl operator to the second equation of (8), in view of the presence of the Coriolis term and the fact that $\operatorname{div} \phi=0$, we deduce that $\phi_{2}=0$ in $\widetilde{\omega} \times(0, T)$ and $\phi_{1}$ is a constant in $\widetilde{\omega} \times(0, T)$. 
Let us introduce $\psi=\partial \phi / \partial x_{1}$ and $\pi=\partial \theta / \partial x_{1}$. We have

$$
\left\{\begin{array}{l}
\psi_{t}-\Delta \psi+\psi+\left(1+x_{2}\right) k \wedge \psi+\nabla \pi=0 \quad \text { in } \quad Q \\
\operatorname{div} \psi=0 \text { in } Q \\
\psi=0 \text { in } \widetilde{\omega} \times(0, T) \\
(\psi, \pi) \in L^{2}(0, T ; H) \times \mathcal{D}^{\prime}(Q)
\end{array}\right.
$$

From the uniqueness property in [4], one has that $\psi \equiv 0$ in $Q$. Since $\partial \phi_{i} / \partial x_{1} \equiv 0, i=1,2$, and $\operatorname{div} \phi=0$ in $Q$, we have $\nabla \phi_{2}=0$ in $Q$, and from $\phi_{2}=0$ in $\widetilde{\omega} \times(0, T)$, we deduce that $\phi_{2} \equiv 0$ in $Q$. On the other hand, since $\partial \phi_{1} / \partial x_{1}=0$ in $Q$ and $\phi_{1}=0$ on $\Sigma_{1}$, we obtain $\phi_{1}=0$ in $C_{1} \times(0, T)$. The uniqueness properties in [3] give $\phi_{1} \equiv 0$ in $Q$, as desired.

Remark 1 In [3], appropriate unique continuation properties have been deduced for the Stokes system, where the main assumption is that all components of the velocity except one vanish in an open non-empty subset of $Q$. At present, we do not know whether a similar result remains true for $(8)$.

The unique continuation property contained in the statement of Proposition 2.2 is equivalent to Theorem 1.2. This proves our first main result. On the other hand, Theorem 1.3 follows from the following observability estimates:

Proposition 2.3 There exist constants $M, C>0$ depending on $\Omega, \omega, \mathcal{O}$ and $T$ such that the following inequality holds true

$$
\int_{0}^{T} \int_{\Omega} \exp \left(-M / t^{4}\right) z^{2} d x d t \leqslant C \int_{0}^{T} \int_{\omega} z^{2} d x d t
$$

for every solution of (8) with $\phi_{0} \in V$.

The proof of Proposition 2.3 relies on certain global Carleman estimates for the cascade system (8). More precisely, let us introduce the weight functions

$$
\left\{\begin{array}{l}
\alpha(x, t)=\left(e^{2 \lambda\left\|\eta_{0}\right\|_{\infty}}-e^{\lambda \eta_{0}}\right) t^{-4}(T-t)^{-4}, \quad \hat{\alpha}(t)=\min _{\bar{\Omega}} \alpha(x, t), \\
\varphi(x, t)=\left(e^{\lambda \eta_{0}}\right) t^{-4}(T-t)^{-4}, \quad \hat{\varphi}(t)=\max _{\bar{\Omega}} \varphi(x, t),
\end{array}\right.
$$

where $\eta_{0} \in \mathcal{C}^{4}(\bar{\Omega})$ is a function satisfying:

$$
\eta^{0}>0 \text { in } \Omega, \quad \eta^{0}=0 \text { on } \Gamma, \quad\left|\nabla \eta^{0}\right|>0 \text { in } \Omega \backslash(\overline{\omega \cap \mathcal{O}}) .
$$

The existence of such a function $\eta^{0}$ is guaranteed in [5].

Proposition 2.4 Assume (5). There exists $\hat{s}=\hat{\sigma}(\Omega, \omega, \mathcal{O})\left(T^{4}+T^{10}\right)$, with $\hat{\sigma}>0$, such that for all $s>\hat{s}$ and for all $\hat{\gamma}<1$ there exist $\hat{\lambda}>1, \hat{\beta}>1$, and $C>0$ such that for any $\lambda>\hat{\lambda}, \beta>\hat{\beta}$, and $\gamma<\hat{\gamma}$, one has:

$$
\left\{\begin{array}{c}
\int_{0}^{T} \int_{\Omega} e^{-2 s \alpha}\left(\frac{1}{s \varphi}\left(|\Delta z|^{2}+\left|z_{t}\right|^{2}\right)+s \lambda^{2} \varphi|\nabla z|^{2}+s^{3} \lambda^{4} \varphi^{3}|z|^{2}\right) d x d t \\
\leqslant C \int_{0}^{T} \int_{\omega} e^{-(1+\gamma) s \hat{\alpha}} \hat{\varphi}^{\beta}|z|^{2} d x d t
\end{array}\right.
$$

for every solution of (8) with $\phi_{0} \in V$, where the constant $C$ depends on $\Omega, \omega, \mathcal{O}$ and $T$, but is independent of $(z, r, \phi, \theta)$. 
Sketch of the proof: We first write the global Carleman inequalities for the Stokes equations given in $[7,6]$ for both systems in (8). The task is then to estimate the integral of $\phi$ in terms of $z$. To this end, the main idea is to follow the steps of the proof of proposition 2.2 in the reversed order. Thus, we estimate $\phi_{1}$ in terms of $\nabla \phi_{1}$, then $\nabla \phi_{1}$ in terms of $\phi_{2}$, then $\phi_{2}$ in terms of $\operatorname{curl} \phi$ and, finally, curl $\phi$ in terms of $z$. At each step, the weight increases. At the end, we find an inequality like (11) with some additional terms in the right hand side that can be suppressed using an appropriate compactness-uniqueness argument.

Remark 2 It must be emphasized that the presence of the Coriolis term plays a crucial role in this argument. It is not clear at all whether theorem 1.3 holds for $(1)$ when $f_{0}=\beta=0$.

Acknowledgements: The authors are grateful to Prof. J.-P. Puel for several fruitful discussions about the subject. The first author was partially supoported by D.G.E.S. (Spain), Grants PB981134 and BFM2000-1317. The second author was supported by FONDAP in Applied Mathematics and by CONICYT (Chile). The third author was partially supported by FONDAP in Applied Mathematics, FONDECYT 1030808 and ECOS C01E02 grants (Chile).

\section{References}

[1] Bodart O., FABre C., Controls insensitizing the norm of the solution of a semilinear heat equation, J. Math. Anal. and Appl. 195 (1995) 658-683.

[2] Bodart O., González-Burgos M., PÉrez-García R., Insensitizing controls for a semilinear heat equation with a superlinear nonlinearity, C. R. Acad. Sci. Paris, Série I 335 (2002) 677-682.

[3] FABRE C., Uniqueness results for Stokes equations and their consequences in linear and nonlinear control problems, ESAIM: COCV 1 (1996) 267-302.

[4] Fabre C., Lebeau G., Régularité et unicité pour le problème de Stokes, Comm. PDE (to appear).

[5] Fursikov V.A., Imanuvilov O.Y., Controllability of Evolution Equations, Lecture Notes, Research Institute of Mathematics, Seoul National University, Korea, 1996.

[6] Guerrero, S., Puel, J.P., to appear.

[7] ImANUVILOV O.Y., Remarks on the controllability of Navier-Stokes equations, ESAIM: COCV 6 (2001) 39-72.

[8] LiOns J.L., Quelques notions dans l'analyse et le contrôle de systèmes à données incomplètes, Proceedings of the XIth Congress on Differential Equations and Applications/ First Congress on Applied Mathematics, pp. 43-54, Málaga (Spain) 1990.

[9] Marchuk G.I., Agoshkov V.I., Shutyaev V.P., Adjoint Equations and Perturbation Algorithms in Nonlinear Problems, CRC Press, Boca Raton, FL 1996. 
[10] Myers P.G., WeAVER A.J., A diagnostic barotropic finite-element ocean circulation model, J. Atmos. and Ocean Tech. 12 (1995) 511-526.

[11] Temam R., Navier-Stokes Equations, Second Edition, North Holland, Amsterdam 1984.

[12] DE TERESA L., Insensitizing control for a semilinear heat equation, Comm. PDE, 25(1/2) (2000) 39-72. 